\title{
Exponential Sensitivity to Dephasing of Electrical Conduction Through a Quantum Dot
}

\author{
J. Tworzydło, ${ }^{1,2}$ A. Tajic, ${ }^{1}$ H. Schomerus, ${ }^{3}$ P.W. Brouwer, ${ }^{4}$ and C.W. J. Beenakker ${ }^{1}$ \\ ${ }^{1}$ Instituut-Lorentz, Universiteit Leiden, P.O. Box 9506, 2300 RA Leiden, The Netherlands \\ ${ }^{2}$ Institute of Theoretical Physics, Warsaw University, Hoża 69, 00-681 Warsaw, Poland \\ ${ }^{3}$ Max-Planck-Institut für Physik komplexer Systeme, Nöthnitzer Str. 38, 01187 Dresden, Germany \\ ${ }^{4}$ Laboratory of Atomic and Solid State Physics, Cornell University, Ithaca, New York 14853-2501, USA
}

(Received 20 July 2004; published 29 October 2004)

\begin{abstract}
According to random-matrix theory, interference effects in the conductance of a ballistic chaotic quantum dot should vanish $\propto\left(\tau_{\phi} / \tau_{D}\right)^{p}$ when the dephasing time $\tau_{\phi}$ becomes small compared to the mean dwell time $\tau_{D}$. Aleiner and Larkin have predicted that the power law crosses over to an exponential suppression $\propto \exp \left(-\tau_{E} / \tau_{\phi}\right)$ when $\tau_{\phi}$ drops below the Ehrenfest time $\tau_{E}$. We report the first observation of this crossover in a computer simulation of universal conductance fluctuations. Their theory also predicts an exponential suppression $\propto \exp \left(-\tau_{E} / \tau_{D}\right)$ in the absence of dephasing - which is not observed. We show that the effective random-matrix theory proposed previously for quantum dots without dephasing explains both observations.
\end{abstract}

DOI: 10.1103/PhysRevLett.93.186806

An instructive way to classify quantum interference effects in mesoscopic conductors is to ask whether they depend exponentially or algebraically on the dephasing time $\tau_{\phi}$. The Aharonov-Bohm effect is of the former class, while weak localization (WL) and universal conductance fluctuations (UCF) are of the latter class [1,2]. It is easy enough to understand the difference: on the one hand, Aharonov-Bohm oscillations in the magnetoconductance of a ring require phase coherence for a certain minimal time $t_{\min }$ (the time it takes to circulate once along the ring), which becomes exponentially improbable if $\tau_{\phi}<t_{\min }$. On the other hand, WL and UCF in a disordered quantum dot originate from multiple scattering on a broad range of time scales, not limited from below, and the superposition of exponents with a range of decay rates amounts to a power law decay.

In a seminal paper [3], Aleiner and Larkin have argued that ballistic chaotic quantum dots are in a class of their own. In these systems the Ehrenfest time $\tau_{E}$ introduces a lower limiting time scale for the interference effects, which are exponentially suppressed if $\tau_{\phi}<\tau_{E}$. The physical picture is that electron wave packets in a chaotic system can be described by a single classical trajectory for a time up to $\tau_{E}$ [4]. Both WL and UCF, however, require that a wave packet splits into partial waves which follow different trajectories before interfering. Only the fraction $\exp \left(-\tau_{E} / \tau_{\phi}\right)$ of electrons which have not yet dephased at time $\tau_{E}$ can therefore contribute to WL and UCF.

The WL correction $\Delta G=\langle G\rangle-G_{\mathrm{cl}}$ is the deviation of the ensemble averaged conductance $\langle G\rangle$ (in zero magnetic field) from the classical value $G_{\mathrm{cl}}=N / 2$. (We measure conductances in units of $2 e^{2} / h$ and assume an equal number of modes $N \gg 1$ in the two leads that connect the quantum dot to electron reservoirs.) The WL correc-
PACS numbers: 73.23.-b, 03.65.Yz, 05.45.Mt, 73.63.Kv

tion according to random-matrix theory (RMT),

$$
\Delta G_{\mathrm{RMT}}=-\frac{1}{4}\left(1+\tau_{D} / \tau_{\phi}\right)^{-1}
$$

has a power law suppression $\propto \tau_{\phi} / \tau_{D}$ when $\tau_{\phi}$ becomes smaller than the mean dwell time $\tau_{D}$ in the quantum dot [5]. Similarly, RMT predicts for the UCF a power law suppression $\propto\left(\tau_{\phi} / \tau_{D}\right)^{2}$ of the mean-squared sample-tosample conductance fluctuations [5,6],

$$
\mathrm{V} \operatorname{ar} G_{\mathrm{RMT}}=\frac{1}{8 \beta}\left(1+\tau_{D} / \tau_{\phi}\right)^{-2},
$$

with $\beta=2$ (1) in the presence (absence) of a timereversal symmetry-breaking magnetic field.

Aleiner and Larkin have calculated the $\tau_{E}$ dependence of the WL correction, with the result [3]

$$
\Delta G=e^{-\tau_{E} / \tau_{\phi}} e^{-2 \tau_{E} / \tau_{D}} \Delta G_{\mathrm{RMT}}
$$

The two exponential suppression factors in Eq. (3) result from the absence of interfering trajectories for times below $\tau_{E}$. The first factor $\exp \left(-\tau_{E} / \tau_{\phi}\right)$ accounts for the loss by dephasing and the second factor $\exp \left(-2 \tau_{E} / \tau_{D}\right)$ accounts for the loss by escape into one of the two leads. The UCF are expected to be suppressed similarly.

The physical picture presented by Aleiner and Larkin is simple and supported by two independent calculations $[3,7]$. And yet, it has been questioned as a result of some very recent computer simulations of UCF $[8,9]$ and WL [10] in the absence of dephasing. The expected exponential reduction of quantum interference effects due to escape into the leads was not observed. In fact, both WL and UCF were found to be completely independent of $\tau_{E}$, even though the simulations extended to system sizes for which $\tau_{E}$ was well above $\tau_{D}$. To explain these 
negative results, Jacquod and Sukhorukov [8] invoked the effective RMT of Silvestrov et al. [11]. In that approach, the electrons with dwell times $>\tau_{E}$ are described by RMT with an effective number $N_{\text {eff }}=N \exp \left(-\tau_{E} / \tau_{D}\right)$ of modes. Then no $\tau_{E}$ dependence is expected as long as $N_{\text {eff }} \gg 1$ - even if $\tau_{E} \gg \tau_{D}$.

Since the predicted exponential reduction factor due to escape into the leads has not appeared in the simulations, it is natural to ask about the factor $\propto \exp \left(-\tau_{E} / \tau_{\phi}\right)$ due to dephasing. Does it exist? An experimental study of twodimensional (2D) weak localization has concluded that it does [12], but since leads play no role in $2 \mathrm{D}$, these experiments cannot really resolve the issue. In the absence of experiments on the zero-dimensional geometry of a quantum dot, we have used computer simulations to provide an answer. We find that a relatively small amount of dephasing is sufficient to introduce a marked $\tau_{E}$ dependence of the UCF. Our observation can be explained by incorporating dephasing into the effective RMT. We find that

$$
\begin{gathered}
\Delta G=e^{-\tau_{E} / \tau_{\phi}} \Delta G_{\mathrm{RMT}}, \\
\mathrm{V} \operatorname{ar} G=e^{-2 \tau_{E} / \tau_{\phi}} \operatorname{Var} G_{\mathrm{RMT}},
\end{gathered}
$$

and show that Eq. (5) provides a fitting-parameter-free description of the numerical data.

We have introduced a dephasing lead [13] in the kicked rotator, which is the same dynamical system studied in Refs. $[8-10,14]$ in the absence of dephasing. The kicked rotator provides a stroboscopic description of chaotic scattering in a quantum dot [15], in the sense that the wave function is determined only at times that are multiples of a time $\tau_{0}$ (which we set to unity). The mean dwell time $\tau_{D}=M / 2 N=\pi / N \delta$ is the ratio of the dimension $M$ of the Floquet matrix (corresponding to a mean level spacing $\delta=2 \pi / M)$ and the dimension $2 N$ of the scattering matrix (without the dephasing lead). The kicking strength $K=7.5$ determines the Lyapunov exponent $\lambda=$ $\ln (K / 2)=1.32$. The Ehrenfest time is given by $[16,17]$

$$
\tau_{E}= \begin{cases}\lambda^{-1} \ln \left(N^{2} / M\right) & \text { if } N>\sqrt{M}, \\ 0 & \text { if } N<\sqrt{M} .\end{cases}
$$

The dephasing lead increases the dimension of the scattering matrix $S$ to $M \times M$. It has the block form

$$
S=\left(\begin{array}{lll}
s_{00} & s_{01} & s_{02} \\
s_{10} & s_{11} & s_{12} \\
s_{20} & s_{21} & s_{22}
\end{array}\right),
$$

where the subscripts 1,2 label the two real leads and 0 labels the dephasing lead. The two real $N$-mode leads are coupled ballistically to the system, while the remaining $M-2 N$ modes are coupled via a tunnel barrier. The dephasing rate $1 / \tau_{\phi}=\Gamma(1-2 N / M)$ is proportional to the tunnel probability $\Gamma$ per mode. The dephasing lead is connected to an electron reservoir at a voltage which is adjusted so that no current is drawn. The conductance $G$ is then determined by the coefficients $G_{i j}=\operatorname{Tr} s_{i j} s_{i j}^{\dagger}$ through Büttiker's formula [13],

$$
\begin{aligned}
G & =G_{12}+\frac{G_{10} G_{02}}{G_{10}+G_{20}} \\
& =G_{12}+\frac{\left(N-G_{11}-G_{12}\right)\left(N-G_{22}-G_{12}\right)}{2 N-G_{11}-G_{12}-G_{22}-G_{21}} .
\end{aligned}
$$

For $\Gamma \ll 1$, the dephasing lead model is equivalent to the imaginary energy model of dephasing [5], which is the model used by Aleiner and Larkin [3]. (We will also make use of this equivalent representation later on.) There exist other models of dephasing in quantum transport $[18,19]$, but for a comparison with Ref. [3], our choice seems most appropriate.

Since we need a relatively small Lyapunov exponent in order to reach a large enough Ehrenfest time, our simulations are sensitive to short nonergodic trajectories. These introduce an undesired dependence of the data on the position of the leads. Preliminary investigations indicated that UCF in a magnetic field was least sensitive to the lead positions, so we concentrate on that quantum interference effect in the numerics. The variance $\operatorname{Var} G$ of the conductance was calculated in an ensemble created by sampling 40 values of the quasienergy. To determine the dependence on the lead positions, we repeated the calculation for 40 different configurations of the leads. Error bars in the plots give the spread of the data.

There are four time scales in the problem: $\lambda^{-1}, \tau_{D}, \tau_{\phi}$, and $\tau_{E}$. To isolate the $\tau_{E}$ dependence we increase both $M$ and $N$ at constant ratio $M / N$ and fixed $K, \Gamma$. Then only $\tau_{E}$ varies. Results are shown in Fig. 1. The variance of the conductance is divided by the RMT prediction (2), with $\beta=2$ because of broken time-reversal symmetry [20]. We see that for $\tau_{\phi} \gg \tau_{E}$, there is no systematic dependence of UCF on the Ehrenfest time, consistent with Refs. [8,9]. However, an unambiguous $\tau_{E}$ dependence appears for $\tau_{\phi} \lesssim \tau_{E}$, regardless of whether $\tau_{\phi}$ is smaller or larger than $\tau_{D}$.

To explain the data in Fig. 1 we introduce dephasing into the effective RMT. For that purpose, it is more convenient to use an imaginary energy than a dephasing lead, so we first make the connection between these two equivalent representations. There exists an exact correspondence for any $N$ [5], which requires a reinjection step to ensure current conservation. For the case $N \gg 1$ of interest here, there is a simpler way.

The coefficients $G_{i j}=G_{i j}^{\mathrm{cl}}+G_{i j}^{\mathrm{q}}$ in Eq. (8) consist of a classical contribution $G_{i j}^{\mathrm{cl}}$ of order $N$ plus a (sample specific) quantum correction $G_{i j}^{\mathrm{q}}$ of order unity. The classical contribution is

$$
G_{i j}^{\mathrm{cl}}=\frac{1}{2} N\left(1+\tau_{D} / \tau_{\phi}\right)^{-1}, \quad \text { for } i, j \in\{1,2\} .
$$



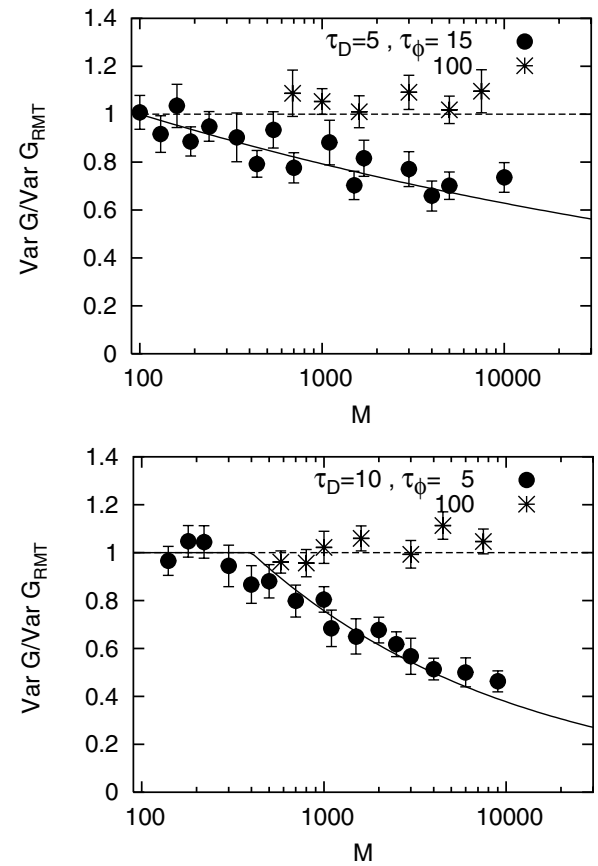

FIG. 1. Variance of the conductance fluctuations, normalized by the RMT value (2), as a function of the dimension $M$ of the scattering matrix of the kicked rotator with a dephasing lead. Each data set is for a fixed value of the dwell time $\tau_{D}=M / 2 N$ and dephasing time $\tau_{\phi}=\Gamma^{-1}(1-2 N / M)^{-1}$. The Lyapunov exponent $\lambda=1.32$ is kept the same for all data sets. The curves show the Ehrenfest time dependence (5) predicted by the effective RMT, without any fit parameter.

Substitution into Eq. (8) gives a classical conductance $G_{\mathrm{cl}}=N / 2$ independent of dephasing - as it should be. To leading order in $N$ we obtain the quantum correction to the conductance,

$$
G_{\mathrm{q}}=\frac{1}{4}\left(G_{12}+G_{21}-G_{11}-G_{22}\right)
$$

(Notice that the classical contribution drops out of the right-hand side.) For $\Gamma \ll 1$, the effect of the dephasing lead on the coefficients $G_{i j}$ is equivalent to the addition of an imaginary part $i \hbar / 2 \tau_{\phi}$ to the energy. With the help of Eq. (10) we can compute the effect of dephasing on WL and UCF,

$$
\begin{gathered}
\Delta G=\left\langle G_{\mathrm{q}}\left(E+i \hbar / 2 \tau_{\phi}\right)\right\rangle, \\
\operatorname{Var} G=\left\langle\left[G_{\mathrm{q}}\left(E+i \hbar / 2 \tau_{\phi}\right)\right]^{2}\right\rangle-(\Delta G)^{2},
\end{gathered}
$$

by averaging the scattering matrix at a complex energy without having to enforce current conservation.

Effective RMT [11] is a phenomenological decomposition of the scattering matrix $S(t)$ in the time domain into a classical deterministic part $S_{\mathrm{cl}}$ for $t<\tau_{E}$ and a quantum part $S_{\mathrm{q}}$ with RMT statistics for $t>\tau_{E}$,

$$
S(t)= \begin{cases}S_{\mathrm{cl}}(t) & \text { if } t<\tau_{E} \\ S_{\mathrm{q}}(t)=S_{\mathrm{RMT}}\left(t-\tau_{E}\right) & \text { if } t>\tau_{E}\end{cases}
$$

The RMT part $S_{\mathrm{q}}$ couples to a reduced number $N_{\text {eff }}=$ $N \exp \left(-\tau_{E} / \tau_{D}\right)$ of channels in each lead. The mean dwell time in the quantum dot of these channels is $\tau_{E}+\tau_{D}$. The classical part $S_{\mathrm{cl}}$ couples to the remaining $2\left(N-N_{\mathrm{eff}}\right)$ channels. (See Ref. [17] for an explicit construction of $S_{\mathrm{cl} .}$.)

Only $S_{\mathrm{q}}$ contributes to $G_{\mathrm{q}}$. Fourier transformation to the energy domain gives

$$
S_{\mathrm{q}}(E)=e^{i E \tau_{E} / \hbar} S_{\mathrm{RMT}}(E)
$$

where we have used that $S_{\mathrm{RMT}}(t)=0$ if $t<0$. The matrix $S_{\mathrm{RMT}}$ has the RMT statistics of a fictitious chaotic cavity with zero Ehrenfest time, $N_{\text {eff }}$ modes in each lead, and the same mean dwell time $\tau_{D}$ as the real cavity (see Fig. 2). For real energy the phase factor $\exp \left(i E \tau_{E} / \hbar\right)$ is irrelevant, hence all $\tau_{E}$ dependence is hidden in $N_{\text {eff }}$ and $\delta_{\text {eff }}$. Since $\Delta G$ and $\operatorname{Var} G$ are independent of these two parameters, they are also independent of $\tau_{E}$. The imaginary part $i \hbar / 2 \tau_{\phi}$ of the energy that represents the dephasing introduces a $\tau_{E}$ dependence of $G_{\mathrm{q}} \propto \exp \left(-\tau_{E} / \tau_{\phi}\right)$. Insertion of this factor into Eqs. (11) and (12) yields the results (4) and (5) given in the introduction.

The curves in Fig. 1 follow from Eq. (5). They describe the simulation quite well-without any fit parameter. To test the agreement between simulation and effective RMT in a different way, we have collected all our data in Fig. 3 in a plot of $-\left(\tau_{\phi} / 2\right) \ln \left(\operatorname{Var} G / \operatorname{Var} G_{\mathrm{RMT}}\right)$ versus $\ln \left(N^{2} / M\right)$. According to Eq. (5), this should be a plot of $\tau_{E}$ versus $\ln \left(N^{2} / M\right)$, which in view of Eq. (6) is a straight line with slope $1 / \lambda=0.76$. There is considerable scatter

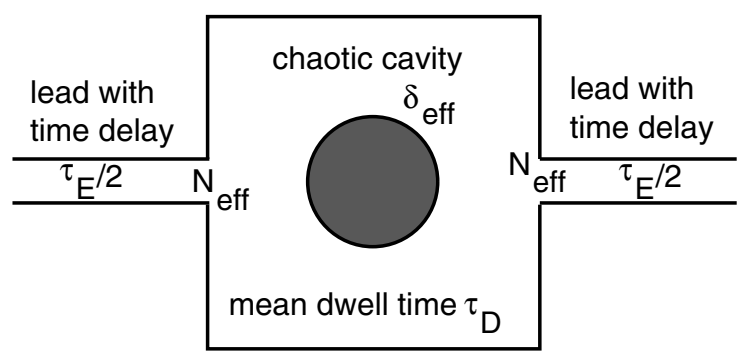

FIG. 2. Pictorial representation of the effective RMT of a ballistic chaotic quantum dot. The part of phase space with dwell times $>\tau_{E}$ is represented by a fictitious chaotic cavity (mean level spacing $\delta_{\text {eff }}$ ), connected to electron reservoirs by two long leads ( $N_{\text {eff }}$ propagating modes, one-way delay time $\tau_{E} / 2$ for each mode). The effective parameters are determined by $N_{\text {eff }} / N=\delta / \delta_{\text {eff }}=\exp \left(-\tau_{E} / \tau_{D}\right)$. The scattering matrix of lead plus cavity is $\exp \left(i E \tau_{E} / \hbar\right) S_{\mathrm{RMT}}(E)$, with $S_{\mathrm{RMT}}(E)$ distributed according to RMT. A finite dephasing time $\tau_{\phi}$ is introduced by the substitution $E \rightarrow E+i \hbar / 2 \tau_{\phi}$. The part of phase space with dwell times $<\tau_{E}$ has a classical scattering matrix, which does not contribute to quantum interference effects. 


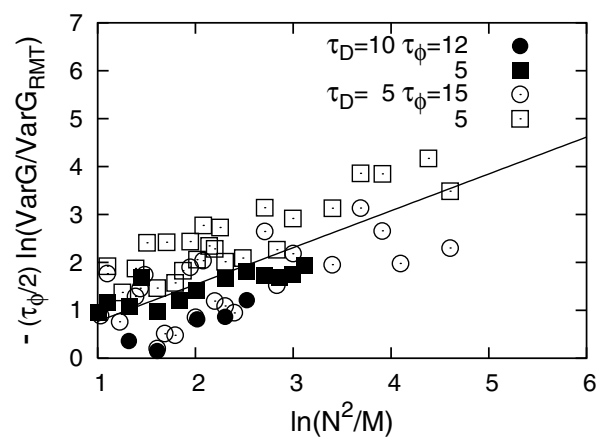

FIG. 3. Four data sets of fixed $\tau_{\phi}, \tau_{D}$, each consisting of a range of $M$ between $10^{2}$ and $2 \times 10^{4}$, plotted on a doublelogarithmic scale. The solid line with slope $1 / \lambda=0.76$ is the scaling predicted by Eqs. (5) and (6).

of the data in Fig. 3, but the systematic parameter dependence is consistently described by the theory as $N$ and $M$ vary over 2 orders of magnitude.

In conclusion, our findings explain the puzzling difference in the outcome of previous experimental [12] and numerical [8-10] searches for the Ehrenfest time dependence of quantum interference effects in chaotic systems: the experiments found a dependence while the computer simulations found none. We have identified the absence of dephasing in the simulations as the origin of the difference. By introducing dephasing into the simulation, we recover the exponential $\tau_{E} / \tau_{\phi}$ suppression factor predicted by Aleiner and Larkin [3]. The effective RMT explains why this suppression factor is observed while the exponential $\tau_{E} / \tau_{D}$ suppression factor of Eq. (3) is not.

It remains an outstanding theoretical challenge to provide a microscopic foundation for the effective RMT, or alternatively, to derive Eqs. (4) and (5) from the quasiclassical theory of Refs. [3,7]. One might think that diffraction of a wave packet at the point contacts is the key ingredient that is presently missing from quasiclassics and which would eliminate the exponential $\tau_{E} / \tau_{D}$ suppression factor from Eq. (3). However, our observation of an exponential $\tau_{E} / \tau_{\phi}$ suppression factor suggests otherwise: if diffraction at the edge of the point contacts were the dominant mechanisms by which wave packets are split into partial waves, then the characteristic time scale for the suppression of quantum interference by dephasing would not be different from the mean dwell time $\tau_{D}$.
This work is part of the research program of the Dutch Science Foundation NWO/FOM. J. T. acknowledges support by the European Community's Human Potential Program under Contract No. HPRN-CT-2000-00144, Nanoscale Dynamics. P.W. B. acknowledges support by the Packard Foundation.

[1] Mesoscopic Phenomena in Solids, edited by B. L. Altshuler, P. A. Lee, and R. A. Webb (Elsevier, Amsterdam, 1991).

[2] Y. Imry, Introduction to Mesoscopic Physics (Oxford University, Oxford, 2002).

[3] I. L. Aleiner and A. I. Larkin, Phys. Rev. B 54, 14423 (1996); Chaos Solitons Fractals 8, 1179 (1997).

[4] G. M. Zaslavsky, Phys. Rep. 80, 157 (1981).

[5] P.W. Brouwer and C.W. J. Beenakker, Phys. Rev. B 55, 4695 (1997); 66, 209901(E) (2002).

[6] H. U. Baranger and P. A. Mello, Phys. Rev. B 51, 4703 (1995).

[7] I. Adagideli, Phys. Rev. B 68, 233308 (2003).

[8] Ph. Jacquod and E.V. Sukhorukov, Phys. Rev. Lett. 92, 116801 (2004).

[9] J. Tworzydło, A. Tajic, and C.W. J. Beenakker, Phys. Rev. B 69, 165318 (2004).

[10] J. Tworzydło, A. Tajic, and C. W. J. Beenakker, cond-mat/ 0405122.

[11] P. G. Silvestrov, M. C. Goorden, and C.W. J. Beenakker, Phys. Rev. Lett. 90, 116801 (2003). For a review, see C.W. J. Beenakker, cond-mat/0406018.

[12] O. Yevtushenko, G. Lütjering, D. Weiss, and K. Richter, Phys. Rev. Lett. 84, 542 (2000).

[13] M. Büttiker, Phys. Rev. B 33, 3020 (1986); IBM J. Res. Dev. 32, 63 (1988).

[14] C. Tian, A. Kamenev, and A. Larkin, Phys. Rev. Lett. 93, 124101 (2004).

[15] A. Ossipov, T. Kottos, and T. Geisel, Europhys. Lett. 62, 719 (2003).

[16] M. G. Vavilov and A. I. Larkin, Phys. Rev. B 67, 115335 (2003).

[17] P. G. Silvestrov, M. C. Goorden, and C.W. J. Beenakker, Phys. Rev. B 67, 241301 (2003).

[18] F. Marquardt and C. Bruder, Phys. Rev. Lett. 92, 056805 (2004); Phys. Rev. B 70, 125305 (2004).

[19] A. A. Clerk and A. D. Stone, Phys. Rev. B 69, 245303 (2004).

[20] Time-reversal symmetry was broken by means of the one-kick Floquet operator of Ref. [10]. 